\title{
On the difficulties of present theoretical models to predict the oxidation state of atomic Au adsorbed on regular sites of $\mathrm{CeO}_{2}(111)$
}

Cite as: J. Chem. Phys. 131, 094702 (2009); https://doi.org/10.1063/1.3216102

Submitted: 27 April 2009 . Accepted: 11 August 2009 . Published Online: 03 September 2009

María Marta Branda, Norberto J. Castellani, Ricardo Grau-Crespo, Nora H. de Leeuw, Norge C. Hernandez, Javier F. Sanz, Konstantin M. Neyman, and Francesc Illas

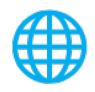

\section{ARTICLES YOU MAY BE INTERESTED IN}

A consistent and accurate ab initio parametrization of density functional dispersion correction (DFT-D) for the 94 elements H-Pu

The Journal of Chemical Physics 132, 154104 (2010); https://doi.org/10.1063/1.3382344

Structure of gold atoms on stoichiometric and defective ceria surfaces

The Journal of Chemical Physics 129, 194708 (2008); https://doi.org/10.1063/1.3009629

Atomic and electronic structure of unreduced and reduced $\mathrm{CeO}_{2}$ surfaces: A first-principles study

The Journal of Chemical Physics 120, 7741 (2004); https://doi.org/10.1063/1.1688316

\section{Lock-in Amplifiers up to $600 \mathrm{MHz}$}
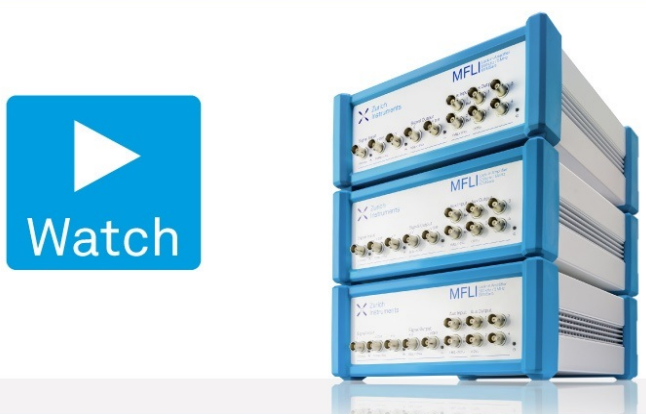

J. Chem. Phys. 131, 094702 (2009); https://doi.org/10.1063/1.3216102 


\title{
On the difficulties of present theoretical models to predict the oxidation state of atomic $\mathrm{Au}$ adsorbed on regular sites of $\mathrm{CeO}_{2}(111)$
}

\author{
María Marta Branda, ${ }^{1}$ Norberto J. Castellani, ${ }^{1}$ Ricardo Grau-Crespo, ${ }^{2}$ \\ Nora H. de Leeuw ${ }^{2}$ Norge C. Hernandez, ${ }^{3}$ Javier F. Sanz, ${ }^{4}$ Konstantin M. Neyman, ${ }^{5,6}$ \\ and Francesc Illas ${ }^{5, a)}$ \\ ${ }^{1}$ Departamento de Física, Universidad Nacional del Sur, 8000 Bahía Blanca, Argentina and Consejo \\ Nacional de Investigaciones Científicas y Tecnológicas (CONICET), Argentina \\ ${ }^{2}$ Department of Chemistry, University College London, 20 Gordon Street, London WC1H OAJ, \\ United Kingdom \\ ${ }^{3}$ Departamento de Física Aplicada I, Escuela Universitaria Politécnica, Universidad de Sevilla, \\ E-41011 Sevilla, Spain \\ ${ }^{4}$ Departamento de Química Física, Facultad de Química, Universidad de Sevilla, E-41012 Sevilla, Spain \\ ${ }^{5}$ Departament de Química Física and Institut de Química Teòrica i Computacional (IQTCUB), \\ Universitat de Barcelona, C/Martí i Franquès 1, 08028 Barcelona, Spain \\ ${ }^{6}$ Institució Catalana de Recerca i Estudis Avançats (ICREA), 08010 Barcelona, Spain
}

(Received 27 April 2009; accepted 11 August 2009; published online 3 September 2009)

\begin{abstract}
The electronic structure and oxidation state of atomic Au adsorbed on a perfect $\mathrm{CeO}_{2}(111)$ surface have been investigated in detail by means of periodic density functional theory-based calculations, using the $\mathrm{LDA}+U$ and $\mathrm{GGA}+U$ potentials for a broad range of $U$ values, complemented with calculations employing the HSE06 hybrid functional. In addition, the effects of the lattice parameter $a_{0}$ and of the starting point for the geometry optimization have also been analyzed. From the present results we suggest that the oxidation state of single $\mathrm{Au}$ atoms on $\mathrm{CeO}_{2}(111)$ predicted by LDA $+U, \mathrm{GGA}+U$, and HSE06 density functional calculations is not conclusive and that the final picture strongly depends on the method chosen and on the construction of the surface model. In some cases we have been able to locate two well-defined states which are close in energy but with very different electronic structure and local geometries, one with Au fully oxidized and one with neutral Au. The energy difference between the two states is typically within the limits of the accuracy of the present exchange-correlation potentials, and therefore, a clear lowest-energy state cannot be identified. These results suggest the possibility of a dynamic distribution of $\mathrm{Au}^{0}$ and $\mathrm{Au}^{+}$atomic species at the regular sites of the $\mathrm{CeO}_{2}(111)$ surface. (C) 2009 American Institute of Physics.
\end{abstract}

[doi:10.1063/1.3216102]

\section{INTRODUCTION}

The discovery of the extraordinary catalytic properties of Au by Haruta ${ }^{1}$ and Goodman and co-workers ${ }^{2}$ triggered extensive research in gold catalysis, with new reactions and new catalysts being reported almost continuously. ${ }^{3,4}$ It is now well established that, although some of the intrinsic properties of gold nanoparticles such as the existence of low coordinated sites $^{5}$ or a critical size for some reactions ${ }^{6,7}$ are largely responsible for the observed chemistry, the catalytic properties of Au-based catalysts are strongly affected by the support. ${ }^{8}$ In particular, Au supported on ceria has been found to be especially active toward the water gas shift (WGS) reaction ${ }^{9,10}$ and has therefore attracted the interest of various groups who examined the catalytic performance of gold nanoparticles supported on ceria for a large set of chemical reactions. ${ }^{11-18}$

The origin of the excellent performance of the $\mathrm{Au} / \mathrm{CeO}_{x}$ catalysts has been investigated by several authors from both experimental and theoretical points of view. One of the most debated issues in this area has been the oxidation state of the

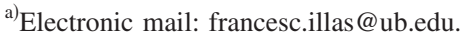

active gold species in the supported catalysts. ${ }^{3}$ Unfortunately, the information currently available appears controversial. Fu et al. ${ }^{10}$ suggested that the high catalytic activity of this system in the WGS reaction arises from positively charged Au, with further evidence indicating that the oxidation state of Au changes with the composition of the gas. ${ }^{19}$ The existence of cationic Au has also been suggested by Guzman et al. ${ }^{17}$ and by Pillai and Deevi ${ }^{20}$ based on data regarding the carbon monoxide oxidation $(\mathrm{CO}+\mathrm{O})$ reaction. Note, however, that the WGS experiments correspond to reducing condition whereas the $\mathrm{CO}+\mathrm{O}$ reaction is performed under oxidizing conditions and, thus, the oxidation state of gold in the catalysis is probably different in both reactions. Recent photoemission experiments ${ }^{21}$ carried out on $\mathrm{Au}$ islands deposited through evaporation on $\mathrm{CeO}_{2}$ thin films showed an $\mathrm{Au}-$ induced reduction from $\mathrm{Ce}^{+4}$ to $\mathrm{Ce}^{3+}$ at the ceria surface, which the authors suggested was associated with the formation of $\mathrm{Au}^{+}$species.

However, x-ray photoelectron spectroscopy experiments on model systems consisting of Au nanoparticles supported on well defined $\mathrm{CeO}_{2}(111)$ seem to indicate that Au nanoparticles remain neutral after the WGS reaction (reducing conditions), which is also consistent with in situ measure- 
ments of near-edge x-ray absorption fine structure for highsurface-area catalysts ${ }^{22-25}$ suggesting that the possible existence of $\mathrm{AuO}_{x}$ species in the as-prepared catalyst ${ }^{17}$ does not correlate with the catalytic activity. According to these results, cationic $\mathrm{Au}$ even if formed will not participate in the active phase. Furthermore, recent experiments carried out under controlled ultrahigh vacuum conditions show that the high activity of the $\mathrm{Au} / \mathrm{CeO}_{x}$ system toward the WGS relies heavily on the capability of the ceria support to dissociate $\mathrm{H}_{2} \mathrm{O}$ molecules. A perfect $\mathrm{CeO}_{2}(111)$ surface is not reactive toward water dissociation, but dissociation takes place as soon as $\mathrm{O}$ vacancies are present. ${ }^{26}$ The presence of reduced ceria is important, but this does not necessarily correlate with the existence of oxidized Au atoms. Gold nanoparticles supported on the $\mathrm{CeO}_{2}(111)$ surface enable water dissociation, allowing the partial reduction in $\mathrm{CeO}_{2}(111)$ by $\mathrm{CO}$ or the $\mathrm{CO} / \mathrm{H}_{2} \mathrm{O}$ mixtures, but without a concomitant oxidation of Au. ${ }^{26}$ Finally, recent experimental work carried out on wellordered ceria films show that atomic Au prefers to be adsorbed on point defects such as step edges. Hence, gold nanoparticles only grow on the $\mathrm{CeO}_{2}(111)$ terraces when the $\mathrm{Au}$ coverage is sufficiently large. ${ }^{27}$ It is worth mentioning that in these experimental studies, gold is forming nanoclusters at the ceria surface. Considering the current debate about the role of $\mathrm{Au}^{\delta+}$ cations in gold catalysis, an experimental investigation of the interaction of individual gold atoms with ceria surfaces would be very desirable.

Next, let us analyze the existing literature concerning the theoretical study of $\mathrm{Au}$ on $\mathrm{CeO}_{2}(111)$, which typically considers the interaction of the surface with individual $\mathrm{Au}$ atoms. These studies are all based on periodic density functional theory (DFT) but use different approximations for the universal but unknown exchange-correlation potential. Unfortunately, this led to some contradictory results in the recent literature. For instance, an earlier work of Liu et al., ${ }^{28}$ based on the use of a generalized gradient approximation (GGA)-type exchange-correlation functional and a modification of the Broyden mixing scheme to preserve broken symmetry solutions arising from the reduction of $\mathrm{Ce}^{4+}$ to $\mathrm{Ce}^{3+}$, suggests that at the atop site of a perfect $\mathrm{CeO}_{2}(111)$ terrace $\mathrm{Au}$ acquires some positive charge leading to a reduced $\mathrm{Ce}^{3+}$ cation. This phenomenon has been reinvestigated by some of the same authors using a GGA $+U$ functional. This later study again concludes that on $\mathrm{CeO}_{2}(111)$ adsorbed $\mathrm{Au}$ is fully oxidized when adsorbed either on top of the oxygen sites or on a bridging site between surface oxygen atoms. ${ }^{29}$ Adsorption at the bridge site being preferred although the difference in adsorption energy between top and bridge sites was 0.21 or $0.13 \mathrm{eV}$ only, for the $2 \times 2$ and $3 \times 5$ supercells, respectively. The bridge site was also found to be the global minimum in the systematic study of the adsorption sites and oxidation state of gold adatoms by Hernandez et al. ${ }^{30}$ These authors further suggested that the stability of the oxidized gold state at the top site could be due to the negative electrostatic potential on top of the surface, while in the case of the bridging site, which was found to be slightly more stable, the charge transfer is also favored by the coordination. However, these results contrast with recent work by Castellani et al. ${ }^{31}$ also using both $\mathrm{LDA}+U$ and $\mathrm{GGA}+U$, suggesting that on that surface $\mathrm{Au}$ tends to remain neutral although it can become oxidized at low-coordination sites modeled by stepped surfaces. These authors also investigated different sites but did not identify the bridge site as the most stable one, probably due to the different computational setup. Here, it is important to point out that Chen et al. ${ }^{32}$ argue that the results obtained with GGA and GGA $+U$ are almost identical, which may be correct for the energy and some related properties but is not necessarily the case for the resulting electronic structure. In fact, these authors did not analyze the net charge on the adsorbed $\mathrm{Au}$ atom, which we consider is the key issue. Clearly a clarification of the charge state of $\mathrm{Au}$ atoms on regular $\mathrm{CeO}_{2}(111)$ surface is urgently needed, which is the aim of the present work.

\section{MODELING CERIA BY MEANS OF DENSITY FUNCTIONAL THEORY}

In order to better understand the origin of the variation in the description of $\mathrm{Au}$ on $\mathrm{CeO}_{2}(111)$ by different DFT-based calculations, it is important to analyze in detail the approaches used in previous studies. First, we note that the standard local density approximation (LDA) and the GGA fail to describe the strongly localized $4 f$ electrons in reduced ceria. This common failure of LDA and GGA to describe strongly correlated systems, not only reduced $\mathrm{CeO}_{x}$, but also compounds as simple as $\mathrm{NiO},{ }^{33}$ usually can be remedied by employing hybrid functionals, which mix a part of exact nonlocal Fock exchange with the local density functional exchange potential. For $\mathrm{CeO}_{2}$ and $\mathrm{Ce}_{2} \mathrm{O}_{3}$, hybrid density functional calculations carried out using either plane wave or Gaussian-type orbitals basis sets ${ }^{34,35}$ led to an accurate description of both systems. In particular, the electronic structure of $\mathrm{Ce}_{2} \mathrm{O}_{3}$ is correctly described with the $4 f$ states well localized on the $\mathrm{Ce}^{3+}$ cations. The hybrid DFT calculations are also able to describe reduced ceria and the particular electronic structure of the resulting $4 f$ localized states. ${ }^{36,37}$ Unfortunately, periodic hybrid DFT calculations face a number of computational problems. Calculations are reasonably fast when using Gaussian-type orbitals (GTOs) but geometry optimization becomes exceedingly slow, because the calculation of energy gradients using GTO becomes the limiting step. The choice of the basis set is also a key question especially for the $4 f$ shell. Geometry optimization is much more efficient when using a plane-wave basis set but here the limiting step is the calculation of the energy with the hybrid functional due to the difficulty in estimating the nonlocal Fock exchange contribution. For bulk solids ${ }^{34,35,38,39}$ or for relatively small unit cells of slab models of a surface, ${ }^{37,40}$ periodic hybrid calculations are possible but computationally expensive. Finally, one must realize that hybrid DFT is not without problems either because the amount of Fock exchange included in the potential is an external input which largely affects the final description. ${ }^{33,41}$

A simple, less computationally demanding, and efficient way to overcome the problems arising from the LDA and GGA exchange-correlated potentials when applied to strongly correlated systems is to correct the self-interaction error by means of a Hubbard-like term by explicit inclusion 
of an effective local two-electron one-center repulsion term $U_{\text {eff }}$ (hereafter referred to simply as $U$ ) resulting in the socalled $\mathrm{LDA}+U$ or GGA $+U$ approaches. ${ }^{42-44}$ The resulting methods allow for a correct description of the electronic structure of reduced ceria. However, the choice of $U$ is a delicate issue for several reasons. First, the physical idea behind this method is to correct the description of the electronic correlation of an electron pair in a given orbital of a given atom provided by LDA or GGA. Clearly, the optimum $U$ values for LDA and GGA can be different. Second, $U$ has to be large enough to lead to well localized $4 f$ electrons but this choice should not generate artificially biased results. Finally, the optimum value of $U$ may depend on the studied property, as evidenced recently by Castleton et al. ${ }^{45}$ Several authors suggest that a correct description of systems based on ceria is achieved by using GGA $+U$ and $U=5 \mathrm{eV}$ and argue that smaller $U$ values will result in artificial delocalization of the $4 f$ electron in reduced ceria. ${ }^{29,46}$ On the other hand, Loschen et al. have suggested that $U$ can be regarded as a semiempirical parameter and have shown that for GGA $+U$ calculations a value of $U=3 \mathrm{eV}$ provides a balanced description of $\mathrm{CeO}_{2}$ and $\mathrm{Ce}_{2} \mathrm{O}_{3}$ whereas a larger value of $U=5 \mathrm{eV}$ is recommended for $\mathrm{LDA}+U$ calculations. ${ }^{47}$ The larger value for LDA is consistent with the fact that GGA will provide a more reliable value of the on-site correlation, although still needing correction. Finally, one has to consider the possibility of using a $U$ value determined in a self-consistent way: 5.30 and $4.50 \mathrm{eV}$ for the $\mathrm{LDA}+U$ and GGA $+U$ methods, respectively. ${ }^{48}$ However, there is no guarantee that a self-consistent $U$ will systematically improve calculated results.

The discussion so far has focused on the DFT method used to compute the energy, electron density, and related properties. There is, however, another difficulty in the DFT description of complex metal oxides, related to the prediction of the equilibrium cell parameters, which is also relevant for the discussion presented in this work. For simple oxides and metals, both LDA and GGA predict lattice parameters in fairly good agreement with experiment. However, for more complex oxides or for transition metals involving heavy atoms, it is known that GGA consistently overestimates interatomic distances, whereas LDA predicts values that are still close to experiment. Therefore, when using GGA functionals to investigate surface properties, one faces a dilemma when choosing the cell parameter to build the surface model. The usual choice is to employ the theoretical equilibrium parameter, where the description of the slab stress is correct, but the geometry can exhibit significant deviation from experiment. An alternative choice is to simply employ the experimental cell parameter, which avoids the artificial cell expansion, although at the expense of introducing a spurious stress on the slab, lateral to the surface, which may lead to unrealistic surface relaxations.

Let us consider the case of $\mathrm{CeO}_{2}$, with an experimental value of the lattice parameter $a_{0} \approx 5.41 \AA$ [5.406(1) $\AA$ (Ref. 49) or 5.411(1) $\AA$ (Ref. 50)]. The GGA-calculated $a_{0}$ is 5.48 $\AA$ (PW91) or $5.47 \AA$ (PBE),$^{34,35,47}$ i.e., larger than the experimental value. This $1.3 \%$ error of the GGA method on $a_{0}$ appears small but it represents a $4.5 \%$ increase in the equi- librium volume. The LDA predicted $a_{0}$ value is $5.36 \AA$, which also represents $1 \%$ error with respect to experiment and will lead to a somewhat compressed lattice. ${ }^{34,47}$ A very accurate $a_{0}$ value results from hybrid DFT calculations: 5.39 and $5.40 \AA$ from plane-wave calculation with the PBE0 and HSE hybrid functionals, respectively, ${ }^{34}$ or $5.41 \AA$ from calculations using a GTO basis set and the HSE functional. ${ }^{35}$ The effect of the $U$ term on the calculated lattice parameter is the same in $\mathrm{LDA}+U$ and $\mathrm{GGA}+U$ schemes: A moderate increase in the calculated $a_{0}$ value with respect to that obtained from standard LDA and GGA $(U=0)$, respectively. Hence, the LDA $+U a_{0}$ value becomes closer to the experiment, increasing to $5.40 \AA$ for $U=5 \mathrm{eV}$, whereas the GGA $+U$ the calculated $a_{0}$ is also increased, thus deviating further from experiment.

At this point one may wonder whether simply using the experimental value for the lattice constant might be preferable. Indeed, this is the usual choice when aiming at predicting properties that strongly depend on the structural parameters, for example, magnetic coupling constants $(J)$ of magnetic oxides such as $\mathrm{NiO} .{ }^{33}$ Here, the use of an experimental lattice parameter allows one to predict values for the magnetic coupling constant which are in very good agreement with experiment, ${ }^{51-53}$ even if the structure is under stress because the forces on the atoms are not vanishing. In this case, the use of an optimized geometry will destroy the agreement with experiment since $J$ varies strongly with the interatomic distance $r$; usually $J \sim r^{-n}$ with $6<n<12$ (see, for instance, Refs. 54 and 55). However, using the experimental geometry for the definition of the unit cell parameter still leaves one with the need to choose a method to relax the surface and to provide the equilibrium geometry of the adsorbed species. In order to overcome this difficulty, in recent studies of stepped ceria surfaces, ${ }^{56}$ ceria nanoparticles, ${ }^{57-59}$ and the adsorption of Au on ceria, ${ }^{31}$ some of us suggested using the $\mathrm{LDA}+U(U=5 \mathrm{eV})$ geometry while employing GGA $+U$ to obtain the energy and the electron density. This approach is not without problems either and difficulties may occur in the calculation of properties for which stress, rather than geometry itself, plays the dominant role. Furthermore, using two different methods for the calculation of geometry and electronic properties could be problematic in situations where a strong coupling between geometry and electronics is to be expected. Indeed, recent work suggested that the calculation of the oxidation state of $\mathrm{Ce}$ atoms in ceria suffers from this difficulty and the nature of the electronic solution depends critically on the assumed geometry or even on the initial geometry for the relaxation. . $^{30,58}$

Having discussed the advantages and drawbacks of the different approaches that can be used to model the properties of ceria surfaces, we should point out that the aim of the present work is not to provide support for any particular choice of methodology, nor to discuss the relative stabilities of surface adsorption sites, but to critically review the effect of the modeling and technical choices on the calculated results and on the emerging physical and chemical pictures of the metal-substrate interaction. In particular, we will demonstrate that the theoretical answer to the problem of the oxidation of atomic gold on the atop site of $\mathrm{CeO}_{2}(111)$ is indeed 
critically affected by the choice of computational approach, including the selection of a $U$ parameter in GGA $+U$ calculations and of the cell parameter used to build the surface model. This will illustrate the limitations of present theoretical methodologies, based on the DFT, in the prediction of the surface chemical properties of complex oxides.

\section{SURFACE MODELS AND COMPUTATIONAL STRATEGY}

The interaction of atomic $\mathrm{Au}$ with the O-terminated $\mathrm{CeO}_{2}(111)$ surface has been studied with the help of a periodic slab with three $\mathrm{O}-\mathrm{Ce}-\mathrm{O}$ layers or a total of nine atomic layers (slab models with only two O-Ce-O layers were employed for the hybrid DFT calculations) and considering mainly the interaction of Au directly on top of one surface $\mathrm{O}$ atom. An earlier study identified this site as the most stable adsorption site for $\mathrm{Au},{ }^{28}$ whereas more recent work ${ }^{29,30}$ suggested that the bridge site is more stable, although the difference in adsorption energy between the two sites is quite small, as commented above. Castellani et al. ${ }^{31}$ also considered a similar bridging site but, with their combined LDA $+U(U=5 \mathrm{eV})$ geometry and GGA $+U(U=3 \mathrm{eV})$ computational setup, this was found to be less stable than the atop site by $\sim 0.1 \mathrm{eV}$. In any case, the main purpose of the present work is to investigate the effect that different choices in the modeling may have on the description of the interaction of $\mathrm{Au}$ with $\mathrm{CeO}_{2}(111)$. In this sense, the consideration of the atop $\mathrm{O}$ site only is justified, since it allows a direct comparison of the different approaches. Nevertheless, selected calculations for the bridge site will also be presented to obtain a more complete description of the interaction of $\mathrm{Au}$ with $\mathrm{CeO}_{2}(111)$

The slab unit cell used in the present work is a $2 \times 2$ simulation cell (Fig. 1), resulting in an Au coverage of 0.25 ML with respect to the number of $\mathrm{O}$ atoms in the surface supercell, with a vacuum width of $\sim 15 \AA$ between the neighboring interleaved slabs along the perpendicular direction to the surface. The slab models were cut from the bulk cubic $(\mathrm{fm} 3 \mathrm{~m})$ fluorite structure using different choices for the lattice parameter, as described below. For the nine atomic layer slab models, the four bottom layers were fixed in positions as calculated for the bulk and the five uppermost layers were allowed to relax fully. The six-layer model used in the more expensive hybrid calculations is derived from the nine layer model, constructed using the corresponding optimized bulk lattice parameter, with the three uppermost layers fully relaxed and the three bottom layers fixed at their geometry from the nine layer slab.

The total energy of the slab model, with or without Au, has been obtained from DFT-based periodic calculations using the $\mathrm{LDA}+U$ and $\mathrm{GGA}+U$ exchange-correlation potentials with different values of $U$ and a basis set with a cutoff of $415 \mathrm{eV}$ for the kinetic energy of the plane waves. The projected augmented wave (PAW) method, ${ }^{60}$ within the implementation of Kresse and Joubert ${ }^{61}$ in the VASP code, ${ }^{62}$ is employed to take into account the effect of the core electrons on the valence density. For completeness, a series of calculations has also been carried out using the HSE06 short (a)

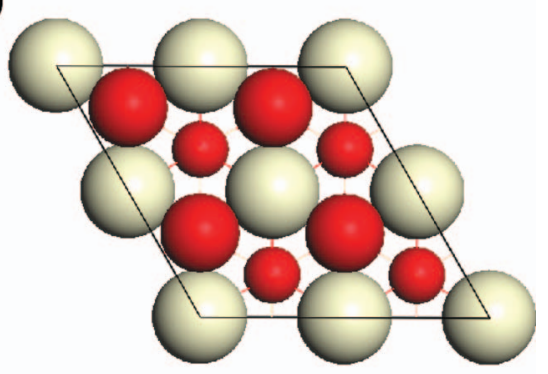

(b)

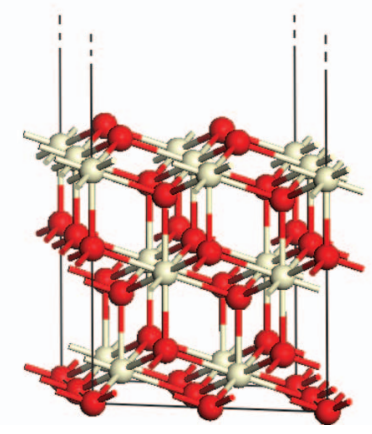

FIG. 1. (a) Top and (b) side views of the $2 \times 2$ unit cell for the $\mathrm{CeO}_{2}(111)$ slab model used in all calculations. Note that the vacuum width between repeated slabs used in the calculations is thicker $(\sim 15 \AA)$ than it appears in the figure.

range (SR) screened hybrid functional. ${ }^{63,64}$ This exchangecorrelation functional takes the form as given in Eq. (1):

$$
\begin{aligned}
E_{x c}^{\mathrm{HSE}}(\omega)= & a E_{x}^{\mathrm{SR}-\mathrm{HF}}(\omega)+(1-a) E_{x}^{\mathrm{SR}-\mathrm{PBE}}(\omega) \\
& +E_{x}^{\mathrm{LR}-\mathrm{PBE}}(\omega)+E_{c}^{\mathrm{PBE}} .
\end{aligned}
$$

It mixes $25 \%$ of exact Fock exchange $(a=0.25)$ in the GGA total energy evaluation but with a value of 0.20 for the $\omega$ screening parameter for the short range. Note that for $\omega$ $\rightarrow 0$ the resulting potential is the PBE0 one, ${ }^{65,66}$ whereas for $\omega \rightarrow \infty$ it leads to the PBE form of the GGA functional. ${ }^{67}$ Inclusion of nonlocal exchange results in a much higher computational complexity when used with a plane wave basis set, which makes full geometry optimization computationally very demanding. Consequently, HSE06 calculations have been carried out only for some special cases. Note that one advantage of hybrid potentials over $\mathrm{GGA}+U$ is that the former modify the whole electronic density and not only the $d$-band (or $f$-band in case of $f$-atoms) of one particular kind of atom. Nevertheless, one must be aware that the hybridDFT based methods are not unambiguous either, since one needs to define the amount of Fock exchange to be used. $^{33,41,68-74}$

The total energy threshold defining self-consistency of the electron density was set to $10^{-4} \mathrm{eV}$ and the convergence criterion for structural optimization was set to be a total energy difference of less than $10^{-2} \mathrm{eV}$ for consecutive geometries. Optimized geometries were then refined until forces on atoms were smaller than $0.01 \mathrm{eV} / \AA^{2}$ and characterized as minimum-energy stationary points by proper analysis of the vibrational frequencies involving the $\mathrm{Au}$ atom. Numerical integration in the reciprocal space was carried out using a $3 \times 3 \times 1$ grid of special $k$-points. ${ }^{75}$ 
In the following section we present series of calculations, which will allow us to separate the influence of different factors on the calculated oxidation state of $\mathrm{Au}$ adatoms at the atop $\mathrm{O}$ sites of the $\mathrm{CeO}_{2}(111)$ surface. For each type of calculation, we analyze the atomic structure, adsorption energy, the magnetic moment, and the Bader ${ }^{76}$ charge. In some of the calculations, adsorbed $\mathrm{Au}$ maintains its atomiclike character, which is evidenced by an almost zero net charge and a magnetic moment of $\approx 0.3 \mu_{B}$. However, in other calculations, $\mathrm{Au}$ becomes oxidized and the signature for the $\mathrm{Au}^{+}$ cationic species is a net charge of $\approx+0.3 e$ and a zero magnetic moment, in agreement with previous work. ${ }^{29-31,77}$ This metal oxidation is accompanied by the appearance of a localized magnetic moment of $\approx 0.9 \mu_{B}$ on one of the surface $\mathrm{Ce}$ atoms, which is a next nearest neighbor (NNN) to the $\mathrm{O}$ atop site, thus indicating its reduction to a $\mathrm{Ce}^{3+}$ state.

\section{RESULTS AND DISCUSSION}

\section{A. Single-point calculations on previously optimized structures corresponding to $\mathrm{Au}^{0} / \mathrm{Ce}^{4+}$ and $\mathrm{Au}^{+} / \mathrm{Ce}^{3+}$ situations: The geometry bias}

We performed a series of single-point calculations on two previously obtained optimized geometries using GGA $+U$ with $U=0,3$, and $5 \mathrm{eV}$ (denoted as G0, G3, and G5, respectively) and $\mathrm{LDA}+U$ with $U=5 \mathrm{eV}$ (L5). The first geometry, from the work of Castellani et al. ${ }^{31}$ was obtained at the L5 level, with the cell parameter corresponding to the L5 equilibrium $\left(a_{0}=5.40 \AA\right)$. The resulting electronic structure (with this setup) corresponds to a neutral state of gold adatom and a concomitant unreduced surface $\left(\mathrm{Au}^{0} / \mathrm{Ce}^{4+}\right.$ situation). The second geometry, from the work of Hernandez et al. ${ }^{30}$ was obtained at the G5 level, with the cell parameter corresponding to the G5 equilibrium $\left(a_{0}=5.49 \AA\right)$. The electronic structure emerging from this setup corresponds to a charge-transfer situation, where gold is $\mathrm{Au}^{+}$and one of the $\mathrm{Ce}$ surface cations (next-nearest neighbor to the $\mathrm{O}-\mathrm{Au}$ bond) is reduced to $\mathrm{Ce}^{3+}$. These two series of calculations will allow us to separate the effect of the initial geometry from that of the method employed in the final energy and electronic structure calculation. Note also that the first geometry corresponds to a high-symmetry structure, which tends to favor an electronic solution where the $\mathrm{Au}$ is neutral, while the second geometry involves a lower-symmetry structure, which favors a solution where the Au is oxidized. The two different solutions may be regarded as electronic states dominated by the neutral or ionic valence bond components, respectively.

We will describe each set of calculations separately. The results for the first series of calculations, performed with the geometry favorable to neutral gold and unreduced ceria, are summarized in Table I. For completeness, this table also reports the results obtained using the L5 methodology. We already mentioned that the $a_{0}$ predicted at the L5 level (5.40 $\AA$ ) almost coincides with the experimentally determined value. It is immediately clear from the results in Table I that the single-point calculations performed at this geometry using $\mathrm{GGA}+U$ calculations all lead essentially to the same result: $\mathrm{Au}$ remains neutral and the ceria surface remains unreduced, that is, no charge transfer takes place between the
TABLE I. Adsorption energy ( $E_{\text {ads }}$ in eV), Bader charge of $\mathrm{Au}\left(q_{\mathrm{Au}}\right.$ in a.u.), and magnetic moments on $\mathrm{Au}$ and $\mathrm{Ce}\left(\mu_{\mathrm{Au}}\right.$ and $\mu_{\mathrm{Ce}}$ in $\left.\mu_{B}\right)$ for the interaction of atomic $\mathrm{Au}$ with the $\mathrm{O}$ top site of the regular $\mathrm{CeO}_{2}(111)$ surface as predicted from single-point calculations at the geometry reported in Ref. 31. (the lattice parameter $a_{0}=5.40 \AA$, the $\mathrm{Au}-\mathrm{O}$ bond length $d\left(\mathrm{Au}-\mathrm{O}_{1}\right)$ $=2.07 \AA$, and the $\mathrm{Ce}-\mathrm{O}$ bond distance in the unrelaxed $\mathrm{CeO}_{2}$ layers equals $2.34 \AA$ ), which is favorable for the neutral gold atom and unreduced ceria. The notations $\mathrm{G} n$ and L5 specify $\mathrm{GGA}+U(U=n \mathrm{eV})$ and $\mathrm{LDA}+U$ $(U=5 \mathrm{eV})$ calculations, respectively.

\begin{tabular}{lcccc}
\hline \hline & G0 & G3 & G5 & L5 \\
\hline$E_{\text {ads }}$ & 0.73 & 0.66 & 0.61 & 1.27 \\
$q_{\mathrm{Au}}$ & +0.01 & -0.02 & +0.05 & -0.05 \\
$\mu_{\mathrm{Au}}$ & 0.29 & 0.31 & 0.33 & 0.32 \\
$\mu_{\mathrm{Ce}}$ & $0.08-0.09^{\mathrm{a}}$ & $0.07-0.10^{\mathrm{a}}$ & $0.06-0.10^{\mathrm{a}}$ & $0.07-0.10^{\mathrm{a}}$ \\
\hline \hline
\end{tabular}

${ }^{\mathrm{a} D e l o c a l i z e d ~ o v e r ~ f o u r ~ C e ~ a t o m s . ~}$

surface and the adatom. Varying $U$ in the $\mathrm{GGA}+U$ series does not cause substantial changes; note that the $\mathrm{Au}-\mathrm{O}$ distance is fixed in this series. The adsorption energy decreases from $0.73 \mathrm{eV}$ for $\mathrm{G} 0$ to $0.61 \mathrm{eV}$ for $\mathrm{G} 5$, but the magnetic moment on adsorbed Au remains close to $0.3 \mu_{B}$, the Bader charge on adsorbed $\mathrm{Au}$ is close to zero and the magnetic moment in the $\mathrm{Ce}$ atoms around this $\mathrm{O}$ surface atom is small $\left(\mu(\mathrm{Ce}) \leq 0.10 \mu_{B}\right)$. The L5 calculation, as expected, also gives the same result, i.e., Au keeps its neutral character and it is not oxidized. The calculated value of the adsorption energy becomes considerably larger $(1.27 \mathrm{eV})$ than that obtained using the GGA potential at any of the $U$ values explored. This is not surprising and results from the wellknown tendency of LDA to overestimate adsorption energies. $^{78,79}$

Now, let us consider the results obtained in the second series of calculations, where the geometry is fixed to the situation corresponding to the charge transfer $\left(\mathrm{Au}^{+} / \mathrm{Ce}^{3+}\right)$ situation. In this case, the lattice parameter and geometry relaxation were calculated at the G5 level. ${ }^{30}$ Under these conditions the computed lattice parameter $a_{0}=5.49 \AA$ is noticeably larger than the experimental value $5.41 \AA .{ }^{49,50}$ The results for this series of calculations are summarized in Table II and indicate that, contrary to the results described above, in this case the adsorption energy increases with increasing

TABLE II. Adsorption energy ( $E_{\text {ads }}$ in eV), Bader charge of $\mathrm{Au}\left(q_{\mathrm{Au}}\right.$ in a.u.), and magnetic moments on $\mathrm{Au}$ and $\mathrm{Ce}\left(\mu_{\mathrm{Au}}\right.$ and $\mu_{\mathrm{Ce}}$ in $\left.\mu_{B}\right)$ for the interaction of atomic $\mathrm{Au}$ with the $\mathrm{O}$ top site of the regular $\mathrm{CeO}_{2}(111)$ surface as predicted from single-point calculations at the geometry reported in Ref. 30 [the lattice parameter $a_{0}=5.49 \AA$, the $\mathrm{Au}-\mathrm{O}$ bond length $d\left(\mathrm{Au}-\mathrm{O}_{1}\right)$ $=1.96 \AA$, and the $\mathrm{Ce}-\mathrm{O}$ bond distance in the unrelaxed $\mathrm{CeO}_{2}$ layers equals $2.37 \AA]$, which is favorable for oxidation of the gold atom. The notations $\mathrm{G} n$ and $\mathrm{L} 5$ specify $\mathrm{GGA}+U(U=n \mathrm{eV})$ and $\mathrm{LDA}+U(U=5 \mathrm{eV})$ calculations, respectively.

\begin{tabular}{lcrrr}
\hline \hline & $\mathrm{G} 0$ & $\mathrm{G} 3$ & $\mathrm{G} 5$ & \multicolumn{1}{c}{ L5 } \\
\hline$E_{\mathrm{ads}}$ & 0.26 & 0.60 & 0.96 & 1.37 \\
$q_{\mathrm{Au}}$ & +0.21 & +0.26 & +0.32 & +0.32 \\
$\mu_{\mathrm{Au}}$ & 0.01 & 0.06 & 0.07 & 0.07 \\
$\mu_{\mathrm{Ce}}$ & $0.70^{\mathrm{a}-0.07^{\mathrm{b}}}$ & $0.79^{\mathrm{c}}$ & $0.94^{\mathrm{c}}$ & $0.93^{\mathrm{c}}$ \\
\hline \hline
\end{tabular}

${ }^{\mathrm{a}}$ Localized in one Ce atom.

${ }^{\mathrm{b}}$ Delocalized over three $\mathrm{Ce}$ atoms.

${ }^{c}$ Fully localized on one Ce atom, NNN to the O-Au bond. 
$U$. This trend does not mean that the solution improves with increasing $U$, but merely reflects the fact that the crystal structure and surface geometry have been consistently calculated at the G5 level. The most important difference between the two series of calculations is that the results in Table II suggest that Au has a marked tendency to become oxidized with a concomitant reduction in the underlying ceria substrate, even for $U=0 \mathrm{eV}$. The net charge in the adsorbed Au atom becomes positive and increases with $U$ up to $+0.32 e$ for $U=5 \mathrm{eV}$. This increase in the charge of the adatom is accompanied by a progressive increase in the magnetic moment of one of the neighboring $\mathrm{Ce}$ atoms. Note that in these single-point calculations there is significant distortion of the underlying $\mathrm{CeO}_{2}$ substrate with a local geometry around the adsorbed $\mathrm{Au}$ atom very different from that in the series of calculations listed in Table I. The result corresponding to $U$ $=0$ deserves some additional comments since it is well established that pure GGA is not able to properly describe the electronic nature of reduced ceria. The present results are in agreement with those reported by Liu et al. ${ }^{28}$ obtained using the GGA lattice parameter and a Broyden mixing scheme together with a pure GGA exchange-correlation functional. These authors found that, under these conditions, the interaction with $\mathrm{CeO}_{2}(111)$ oxidizes the adsorbed $\mathrm{Au}$ atoms. Nevertheless, it is important to note that for $U=0$ the oxidation of $\mathrm{Au}$ is accompanied, as expected, by reduction in the underlying substrate but here the electron transferred to the substrate is delocalized among various Ce atoms. Increasing the value of $U$ up to $5 \mathrm{eV}$ enhances the tendency of $\mathrm{Au}$ to lose one electron and results in a completely oxidized $\mathrm{Au}$ atom, but now with a transfer of one electron to one of the $\mathrm{Ce}$ atoms, where the electron is well localized in a $4 f$ orbital, indicating the formation of a $\mathrm{Ce}^{3+}$ species. Apart from the fact that here Au becomes oxidized, which is qualitatively different from the results described for the previous series of calculations, we should emphasize that the surface geometry is markedly different here, with one longer $(3.97 \AA$ ) and one shorter $(3.67 \AA) \mathrm{Ce}-\mathrm{Ce}$ distance, which represents roughly a $0.15 \AA$ expansion/compression of the $3.82 \AA$ value for the relaxed clean surface. This structural distortion is the result of the $\mathrm{Ce}^{4+} \rightarrow \mathrm{Ce}^{3+}$ reduction, since the latter has a larger atomic volume, and therefore $\mathrm{Ce}^{3+}-\mathrm{O}$ distances are larger than $\mathrm{Ce}^{4+}-\mathrm{O}$ distances. The result of a L5 single-point calculation at this geometry is again very similar to the results for $\mathrm{GGA}+U$, showing clear $\mathrm{Au}$ oxidation and Ce reduction, but with a stronger adsorption.

The overall conclusion from the two series of calculations described above is that the level of theory used for a single-point calculation at a given geometry has very little effect on the nature of the electronic solution obtained. In other words, the geometry already contains the information of the character of the electronic state, which does not change when using different values of $U$, provided that further relaxation is not allowed.

\section{B. Full relaxation calculations for different values of the cell parameter}

Having concluded that the geometry largely determines the character of the final solution, we now want to clarify the effect of different aspects of the geometry. The two structures studied above differ not only in the local geometry at the surface but also in the value of the cell parameter. Indeed, one can argue that the effect of the cell parameter itself is very important and that more compressed cells will tend to favor an unreduced ceria surface (as there is no space available for the required increase in size of the Ce cation upon reduction to the $3+$ state), while, conversely, more expanded cells would favor the reduced ceria solution, accompanied by the oxidation of gold. We will therefore try to separate the effect of the cell parameter from that of the local geometry, in order to check whether the difference in cell parameters is enough to explain the results presented in the last subsection. We will do that by performing full relaxation calculations of the atomic coordinates at different fixed values of $a_{0}$. It is worth noting that making the cell parameter an independent variable is not a purely methodological exercise. Small compression or expansion of the surface can in principle be achieved by growing thin films of the oxide material on top of a metal support with different cell parameters. The cell parameter can also be experimentally modified by applying external pressures or by doping the bulk materials.

Three new series of calculations (with G0, G3, and G5) were therefore carried out by fixing the lattice parameter to different values $\left(a_{0}=5.35-5.55 \AA\right.$, in steps of $\left.0.05 \AA\right)$ and relaxing the structures starting from geometries close to those described above for the neutral and oxidized Au adatom. In the set of calculations with $U=0 \mathrm{eV}$, summarized in the top panel of Fig. 2, for all values of the lattice parameter the predicted ground state corresponds to neutral $\mathrm{Au}$, but with a trend to become slightly charged at the larger values of $a_{0}$, although the Bader charge remains well below the value of 0.3 a.u. corresponding to oxidized Au. Changing $U$ from 0 to $3 \mathrm{eV}$ (middle panel of Fig. 2) does not have any significant effect for the smaller values of the cell parameters, around $a_{\exp }$ and below. However, for the value of $a_{0}$ $=5.45 \AA$ and above, a second solution appears, which corresponds to a partially oxidized state of $\mathrm{Au}$, and with a very similar energy to the solution with neutral gold. Thus, when carrying out a full relaxation using the theoretical equilibrium cell parameter at the G3 level, the two solutions coexist and the differences in energy between them are so small that it is no longer possible to reliably identify the ground state; one solution or the other are obtained from the relaxation depending only on the starting structure. The stability of the solution with $\mathrm{Au}^{+}$with respect to the solution with $\mathrm{Au}^{0}$ increases slightly when increasing the cell parameter, but even at the largest value of the lattice parameter investigated here $(5.55 \AA)$, the two solutions remain very close in energy.

For $U=5 \mathrm{eV}$ (bottom panel of Fig. 2) the situation is very different except for the lowest value of the lattice parameter $\left(a_{0}=5.35 \AA\right)$, which is smaller than the experimental one $\left(a_{0}=5.41 \AA\right)$, the ground state corresponds to a fully oxidized Au atom. For $a_{0}=5.35 \AA$ and $a_{0}=5.40 \AA$ two solutions occur, one with and another without $\mathrm{Au}$ oxidation, depending on the initial structure in the geometry optimization. In the first case, the solution corresponding to oxidized $\mathrm{Au}$ is only $55 \mathrm{meV}$ higher in energy, whereas in the second case the solution with neutral $\mathrm{Au}$ is $153 \mathrm{meV}$ higher in en- 

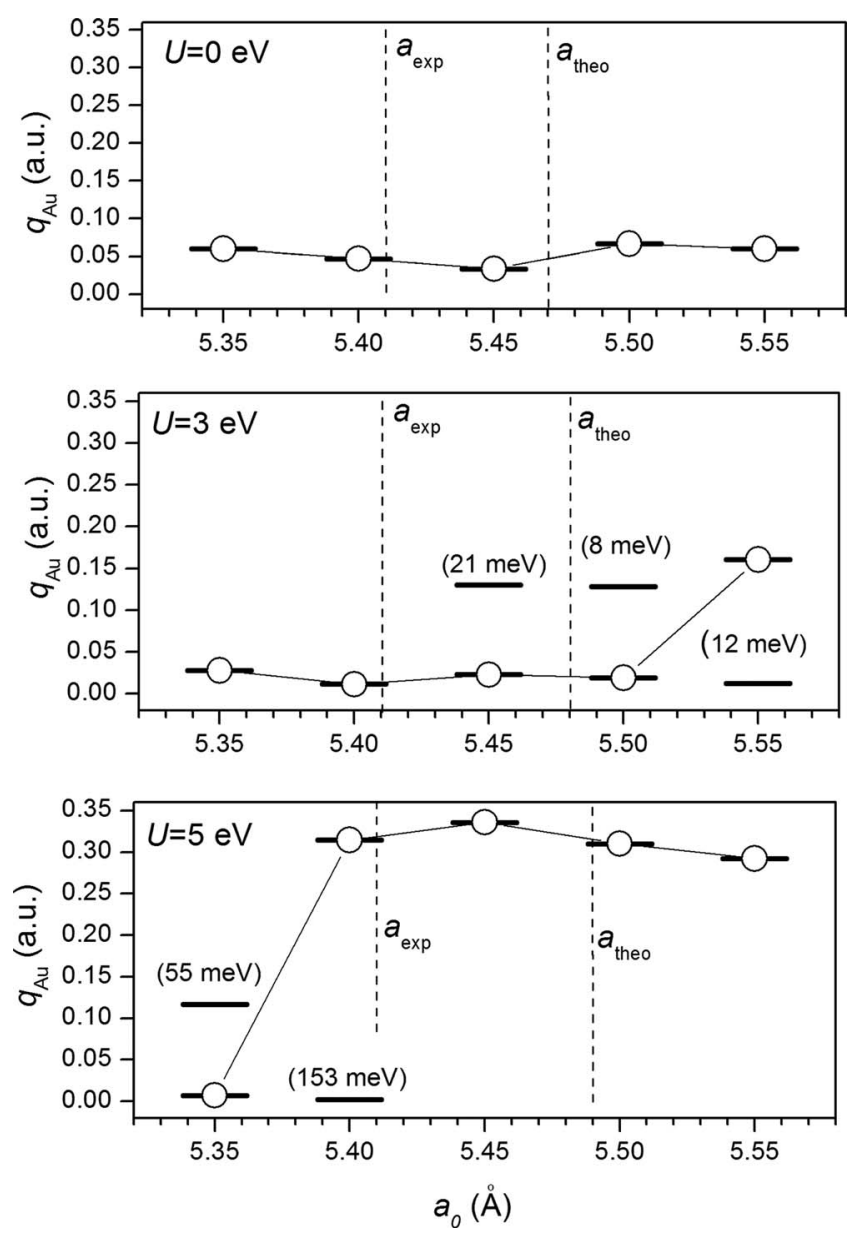

FIG. 2. Effect of lattice parameter and $U$ value on the oxidation state of Au atom adsorbed on the $\mathrm{O}_{1}$ top site of $\mathrm{CeO}_{2}(111)$. Reported on the vertical axis are the Bader charges for adsorbed $\mathrm{Au}$; note that a fully oxidized $\mathrm{Au}$ atom is characterized by a Bader charge of roughly $+0.35 e$. As specified in the text, the calculations were started from geometries calculated for the neutral and oxidized Au adatom. Top, middle, and bottom panels report results for $U=0,3$, and $5 \mathrm{eV}$, respectively. Circles denote the ground state whereas horizontal lines denote an excited configuration at an energy from the ground state given in parentheses. The vertical dashed lines indicate the experimental and calculated (within the corresponding GGA $+U$ method) value of the lattice parameter.

ergy than the one where Au is oxidized. When the calculation is performed with a cell parameter around the theoretical value for the G5 method, only the solution with Au oxidation could be found. As an illustration of the coexistence of the two different types of electronic solutions in some critical cases, we plotted the partial densities of states (DOSs) corresponding to the two solutions with $U=5 \mathrm{eV}$ and $a_{0}$ $=5.40 \AA$ (Fig. 3). The lowest energy solution in this case corresponds to oxidized gold and a reduced surface Ce cation, where the Ce $4 f$ peak is just below the Fermi level and the Au $6 s$ states are empty [Fig. 3(a)]. However, the Ce $4 f$-Au $6 s$ energy separation is so small that the excited state with an $\mathrm{Au}^{0}\left(6 s^{1}\right)$ configuration is very close in energy (153 $\mathrm{meV}$ in this case) to the ground state. This second solution is shown in Fig. 3(b), where the Ce $4 f$ are all above the Fermi level.

Finally, we also performed full relaxation calculations at the L5 level, starting from the two different types of geometry discussed above. In this case, the variation with the cell

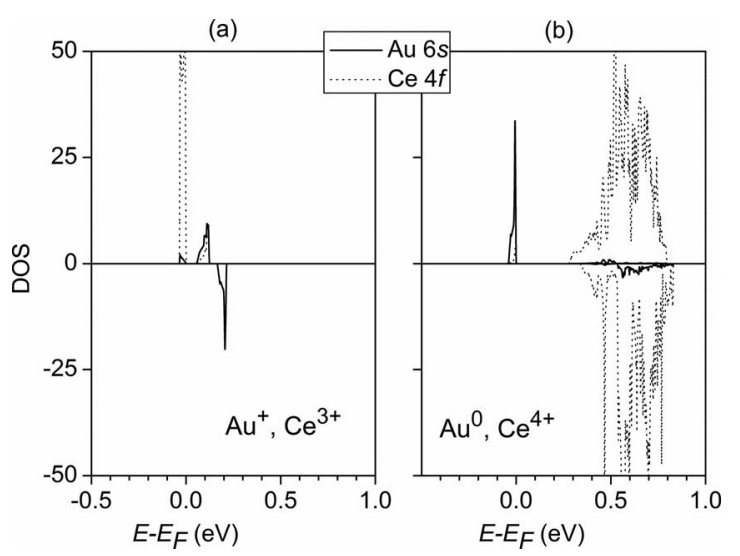

FIG. 3. Electronic DOS projected on the Au $6 s$ and Au $4 f$ orbitals for the two different solutions obtained with GGA $+U(U=5 \mathrm{eV})$ and $a_{0}=5.40 \AA$, corresponding to (a) oxidized $\mathrm{Au}$ and reduced $\mathrm{Ce}$ cation and (b) neutral gold and unreduced $\mathrm{Ce}^{4+}$. Positive and negative values of the DOS correspond to spin up and spin down components, respectively.

parameter was not investigated, as the theoretical equilibrium value for this method $(5.40 \AA)$ is very close to the experimental value. Again, the initial geometry determined the nature of the solution. The lowest-energy case corresponds to oxidized gold and one reduced Ce cation in the next nearest neighbor position to the $\mathrm{O}$ atom of the top site, although the solution without charge transfer, which is the one reported in Ref. 31, is only $51 \mathrm{meV}$ higher.

It is clear from these series of calculations that the GGA $+U$ result on the oxidation state of gold depends both on the value of the cell parameter employed to build the surface slab and on the $U$ value used in the calculation of the local geometry. Larger $U$ values tend to favor the oxidized gold solution, while lower $U$ values tend to give the neutral gold state, which is in line with previous work on the $\mathrm{Pd} /$ ceria system, showing that the introduction of $U$ in the GGA description is necessary to reproduce the experimentally observed charge transfer between Pd adatoms and surface $\mathrm{Ce}$ cations. ${ }^{80}$ Also, the value of the cell parameter critically affects the nature of the solution. When the cell is too compressed, it is energetically unfavorable to accommodate the larger $\mathrm{Ce}^{3+}$ cation, and therefore an unreduced surface is preferred. In general, the larger the cell parameter, the more favorable becomes the charge transfer solution. This observation explains the discrepancy between the results of Castellani et $a l .{ }^{31}$ (G3 at $a_{0} \approx 5.40 \AA$, observing neutral gold), and of Hernandez et $a l^{30}$ and Zhang et al. ${ }^{29}$ (G5 at $a_{0}$ $\approx 5.49 \AA$, reporting oxidized gold). As stated above, we do not intend to argue here which approach corresponds better to the real ceria surface in a relaxed state. However, our results do show clearly that the answer critically depends on the computational approach and model chosen, even if the variations in $U$ (from 3 to $5 \mathrm{eV}$ ) or in the cell parameter (from 5.40 to $5.49 \mathrm{eV}$ ) may appear small.

\section{Calculations with the HSE06 hybrid functional}

The calculations described so far are all based on the $\mathrm{LDA}+U$ or $\mathrm{GGA}+U$ approaches. These methods introduce the $U$ term on the $\mathrm{Ce}(4 f)$ level only and, hence, the main effect on the electron density is precisely on these levels. In 
this sense, a better description may be reached by using hybrid functionals, where the introduction of the nonlocal Fock exchange potential has a direct effect on the whole electron density and not only on the Ce $f$ levels. It should be reiterated, however, that the precise amount of the Fock exchange needed in hybrid functionals [cf. Eq. (1)] cannot be anticipated easily and may be system dependent. ${ }^{33,41,68-74,81}$ Therefore, the amount of Fock exchange included in the functional could affect the prediction in a similar way as the amount of Hubbard correction does in the $\mathrm{LDA}+U$ and $\mathrm{GGA}+U$ calculations.

We now present the results from hybrid DFT calculations within the HSE06 functional. They were carried out first for two fixed geometries using nine atomic layer slab models. Results for this model obtained using the GGA $+U$ approach show that the reduced thickness does not noticeably affect the energy difference between the two solutions. Again, the calculations converged to a solution with oxidized or neutral Au, depending on the nature of the geometry (favorable or unfavorable to oxidation). This result is in line with the conclusions extracted from the LDA $+U$ and GGA $+U$ calculations and confirms that the starting geometry and the lattice parameter play a fundamental role.

Next, calculations were also carried out at the HSE06optimized lattice parameter $(5.40 \AA)$, including relaxation of the surface atoms, but starting from the two different geometries, one close to the solution corresponding to adsorbed neutral $\mathrm{Au}$ and one corresponding to oxidized adsorbed Au. Since these calculations are computationally very demanding, we used here the six layer slab model and kept fixed the three bottom layers. Again, two solutions are found, one with neutral and another with fully oxidized $\mathrm{Au}$. In the latter, the oxidation is accompanied by the $\mathrm{Ce}^{4+} \rightarrow \mathrm{Ce}^{3+}$ reduction of one of the surface $\mathrm{Ce}$ atoms, with a concomitant localization of one electron in the $\mathrm{Ce}(4 f)$ level with a magnetic moment of $\mu(\mathrm{Ce})=0.93$. In this case, the lowest energy solution corresponds to the neutral state of gold, but the energy difference between the two states is not very large, about $150 \mathrm{meV}$.

From the whole set of results described above it is clear that the calculated interaction of the Au with the atop $\mathrm{O}$ site at the perfect $\mathrm{CeO}_{2}(111)$ surface depends on the exchangecorrelation potential used and the model of the substrate surface. One may suggest that the differences encountered when using different approaches have their origin in the proximity of two electronic states. Hence, using one computational setup or another may favor different electronic solutions, resulting in different qualitative pictures of the adsorbed species, at least at $0 \mathrm{~K}$. In most cases, the energy difference is so small that one can safely claim that at higher temperature the two states may coexist and, as a result, a statistical distribution would be present. In some other cases, the energy difference is larger, precluding the occurrence of both solutions.

\section{Comparison with bridge site}

The whole discussion above is based on results obtained from a systematic study where $\mathrm{Au}$ interacts directly with an O surface atom (atop site). This site was reported to be the most stable for $\mathrm{Au}$ adsorption on $\mathrm{CeO}_{2}(111)$ at the $\mathrm{G} 3$ level, with geometry and the cell parameter corresponding to the L5 $\left(a_{0}=5.40 \AA\right)$ and Au remained neutral. ${ }^{31}$ However, as already mentioned, Zhang et $a .^{29}$ and Hernandez et al. ${ }^{30}$ reported that at the G5 level (with $a_{0}=5.49 \AA$ ) the global minimum corresponds to a bridge site where $\mathrm{Au}$ interacts directly with two $\mathrm{O}$ surface atoms and that here $\mathrm{Au}$ becomes oxidized. It seems therefore appropriate to compare at least these two approaches (G3 with $a_{0}=5.40 \AA$ and G5 with $a_{0}$ $=5.49 \AA$ ) for the bridge site as well, in each case starting from geometries favorable to neutral or to oxidized $\mathrm{Au}$, similar to our study of the top site. From this additional set of calculations, several important conclusions emerge, which fully confirm the finding of the systematic study described above for the atop site and add also new perspectives.

First, the bridge site behaves differently from the top site. Even with G3 and $a_{0}=5.40 \AA$ (the optimum value for L5 and for hybrid functionals and also very close to experiment), this site is only stable for an electronic structure where $\mathrm{Au}$ is oxidized with a calculated Bader charge of +0.36 a.u. Any attempt to converge to a solution with neutral bridging Au resulted in a final geometry where Au moves either to the top site of to a different type of bridge sites involving one $\mathrm{O}$ and one $\mathrm{Ce}$ surface atom. The latter is the global minimum for these calculation settings and is similar to the most stable adsorption geometry of neutral gold at the isostructural (111) surface of cubic zirconia. ${ }^{82}$ Thus, a very interesting and important result is that the charge transfer (oxidized $\mathrm{Au}$ ) solution at the bridge site is not the global minimum at the G3 (and $a_{0}=5.40 \AA$ ) level. In fact, it has an energy $0.09 \mathrm{eV}$ higher than the neutral Au solution at top site and $0.13 \mathrm{eV}$ higher than the neutral Au solution at the $\mathrm{O}-\mathrm{Ce}$ bridge site.

The comparison between the top and bridge sites allows us to conclude that not only can the nature of the solutions, but also the relative stability of the sites change with the choice of computational parameters. Finally, the reason why the bridge site behaves in a different way from the top site is likely because twofold coordination favors charge transfer allowing a spatial redistribution of the charge, via hybridization, in a way that minimizes the repulsion between the filled orbitals of the gold atoms and those of the surface atoms. ${ }^{30}$

\section{CONCLUSIONS}

The electronic structure and oxidation state of atomic $\mathrm{Au}$ adsorbed on the regular $\mathrm{O}$ sites of the perfect $\mathrm{CeO}_{2}(111)$ terrace has been investigated in detail by means of periodic DFT-based calculations using the $\mathrm{LDA}+U, \mathrm{GGA}+U$, and HSE06 exchange-correlation potentials. For the LDA $+U$ and $\mathrm{GGA}+U$ series of calculations a broad range of $U$ values has been explored. In addition, the effect of the lattice parameter $a_{0}$ and of the geometry optimization procedure has also been analyzed by systematically varying $a_{0}$ and by using different ways to obtain the optimum geometry.

From this systematic study it appears that the final description of the physical and chemical properties is strongly affected by various factors, including the way the slab surface model is constructed and the choice for the starting geometry, but with the exchange-correlation potential playing a 
fundamental role. The LDA $+U$ calculations with $U=5 \mathrm{eV}$ predict two solutions: One with oxidized $\mathrm{Au}$, provided the proper starting geometry is used, and one with a slightly higher energy $(+51 \mathrm{meV})$ where $\mathrm{Au}$ remains neutral. The picture emerging from the GGA $+U$ calculations depends markedly on the value of $U$. For $U=0$ (equaling pure GGA), only solutions with neutral gold were found, while for $U$ $=3 \mathrm{eV}$ the solution depends on the cell parameter employed in the calculation; for values around the experimental cell parameter, a neutral state of the adatom is predicted, but for the (somewhat larger) theoretical equilibrium cell parameter, both types of solution are found, with energies differing by only a few meV. When $U=5 \mathrm{eV}$ the electronic ground state corresponds to oxidized $\mathrm{Au}$ except for unrealistically small values for the lattice parameter. Finally, two electronic states with neutral and oxidized $\mathrm{Au}$ are also found in the hybrid HSE06 calculations, but in this case the energy is lower (by around $150 \mathrm{meV}$ ) for the solution with neutral Au.

A common feature of the calculations is the simultaneous existence of two nearly degenerate solutions, one with neutral and one with fully oxidized adsorbed Au. This finding makes it extremely difficult to reach a firm conclusion about the final oxidation state of $\mathrm{Au}$ adsorbed on the regular $\mathrm{CeO}_{2}(111)$ surface. However, this study does show conclusively that all methods are capable of finding both solutions for reasonable values of the lattice parameter as well as of the $U$ value in the $\mathrm{LDA}+U$ and GGA $+U$ methods or of the amount of Fock exchange in the hybrid DFT calculations and that the two solutions are close in energy. Unfortunately, the energy difference between the two solutions is within the accuracy limits of the present state of the art DFT methods and thus we cannot determine conclusively which solution is the absolute minimum energy situation.

Note also that in the case where $\mathrm{Au}$ is oxidized, the surface atomic structure is noticeably changed due to the need to accommodate the larger $\mathrm{Ce}^{3+}$ cation resulting from the charge transfer between the $\mathrm{Au}$ adatom and the oxide surface. The energy required to accommodate this distortion is probably gained from the attractive electrostatic interaction between the oxidized gold and the oxygen atoms at the surface. As a result, the two types of solutions exhibit similar energies and any small change in the calculation methodology changes the nature of the ground state.

To summarize, the oxidation state of $\mathrm{Au}$ on $\mathrm{CeO}_{2}(111)$ predicted by $\mathrm{LDA}+U, \mathrm{GGA}+U$ and hybrid HSE06 density functional calculations is still uncertain and strongly depends on the method chosen, although the starting geometry and the way the surface model is constructed also play a role. Rigorously speaking, it is not possible to reach a firm and definitive answer and the final picture depends on which of the used methods has more credit for the reader. Some will argue that the GGA $+U$ exchange-correlation potential is accurate enough and claim that hybrid functionals may introduce spurious effects and some will argue the contrary, namely, that hybrid functionals have proven to provide numerical results in better agreement with experiment and to properly describe strongly correlated systems. It is likely that the answer to this dilemma can only be found by especially careful surface science experiments. Nevertheless, all meth- ods agree in predicting a very small energy difference between the two types of solution for the description of $\mathrm{Au}$ interacting with the $\mathrm{O}$ atop site.

Selected calculations carried out for the bridge site show that the relative stability of the sites can change with the choice of computational parameters. The global minimum predicted by the G3 setup with $a_{0}=5.40 \AA$ is close to the atop site but with $\mathrm{Au}$ forming an angle of $28^{\circ}$ with respect to the perpendicular to the surface and with Au remaining neutral. At this level adsorption at the bridge site leads to a solution with oxidized Au but $0.13 \mathrm{eV}$ above the global minimum. This result is different from the prediction arising from G5 with $a_{0}=5.49 \AA$, where $\mathrm{Au}$ is oxidized and the global minimum is the bridge site with an energy $0.13 \mathrm{eV}$ lower than for the atop site. The systematic analysis carried out in this work for the atop site allows us to better understand the origin of these differences and the determining influence of the choice of some computational parameters.

In any case, the very small energy difference between neutral and cationic Au adsorbed at the surface strongly suggests the presence of a statistical distribution of the two species at the most stable adsorption sites of the $\mathrm{CeO}_{2}(111)$ surface as a function of temperature. Thus, practical applications (especially at elevated temperature) should definitely take into account this unusual and interesting interplay of the $\mathrm{Au}^{0} / \mathrm{Au}^{+}\left(\mathrm{Ce}^{4+} / \mathrm{Ce}^{3+}\right)$ states even in the absence of defects on the $\mathrm{CeO}_{2}(111)$ surface. The close energetic proximity of these neutral and oxidized states of atomic Au adsorbates on ceria is expected to strongly contribute to the observed richness of chemistry of this important type of supported metal systems.

Finally, one must be aware that the difficulties encountered in describing $\mathrm{Au}$ on $\mathrm{CeO}_{2}(111)$ by means of DFT methods are more generally applicable to many strongly correlated systems, for example $\mathrm{NiO}^{33}$ the high-critical temperature superconducting parent compounds, ${ }^{53}$ manganites, ${ }^{70}$ and the new iron pnictides. ${ }^{83}$ It is clearly important in calculations of these types of materials that we check the accuracy of the exchange-correlation functionals against experimental data before extracting definite conclusions from the calculations.

\section{ACKNOWLEDGMENTS}

The authors are indebted to Professor Georg Kresse for making available version 5.1 of VASP with the implementation of the hybrid functional available prior to public distribution. This study has been supported by the CONICETArgentina, Spanish Ministry of Science and Innovation (MICINN) via Grant Nos. FIS2008-02238, MAT200804918, HA2006-0102, and CTQ2007-30547-E/BQU and by the COST-D41 action. Computational time on the MareNostrum supercomputer of the Barcelona Supercomputing Center/Centro Nacional de Supercomputación is gratefully acknowledged. R.G.C. and N.H.d.L. acknowledge support from EPSRC Grant No. EP/C51744X and access to the $\mathrm{HPC}_{x}$ and HECToR high performance computing facilities 
provided by the Materials Chemistry Consortium via EPSRC Grant Nos. EP/D504872/1 and EP/F067496/1. R.G.C. also acknowledges support from Transnational Access grant from the HPC-Europa Project.

${ }^{1}$ M. Haruta Catal. Today 36, 153 (1997); Nature (London) 437, 1098 (2005).

${ }^{2}$ M. Valden, X. Lai, and D. W. Goodman, Science 281, 1647 (1998)

${ }^{3}$ A. S. K. Hashmi and G. J. Hutchings, Angew. Chem. Int. Ed. 45, 7896 (2006).

${ }^{4}$ G. J. Hutchings, M. Brust, and H. Schmidbaur, Chem. Soc. Rev. 37, 1759 (2008), and the series of articles in Issue 9 dedicated to the chemistry of nanogold.

${ }^{5}$ A. Corma, M. Boronat, S. González, and F. Illas, Chem. Commun. (Cambridge) 2007, 3371 (2007)

${ }^{6}$ M. Turner, V. B. Golovko, O. P. H. Vaughan, P. Abdulkin, A. BerenguerMurcia, M. S. Tikhov, B. F. G. Johnson, and R. M. Lambert, Nature (London) 454, 981 (2008).

${ }^{7}$ A. Roldán, S. González, J. M. Ricart, and F. Illas, ChemPhysChem 10, 348 (2008).

${ }^{8}$ M. Boronat, P. Concepción, A. Corma, S. González, F. Illas, and P. Serna, J. Am. Chem. Soc. 129, 16230 (2007).

${ }^{9}$ Q. Fu, A. Weber, and M. Flytzani-Stephanopoulos, Catal. Lett. 77, 87 (2001)

${ }^{10}$ Q. Fu, H. Saltsburg, and M. Flytzani-Stephanopoulos, Science 301, 935 (2003).

${ }^{11}$ Q. Fu, W. Deng, H. Saltsburg, and M. Flytzani-Stephanopoulos, Appl. Catal., B 56, 57 (2005).

${ }^{12}$ D. Andreeva, V. Idakiev, T. Tabakova, L. Ilieva, P. Falaras, A. Bourlinos, and A. Travlos, Catal. Today 72, 51 (2002).

${ }^{13}$ T. Tabakova, F. Boccuzzi, M. Manzoli, and D. Andreeva, Appl. Catal., A 252, 385 (2003).

${ }^{14}$ A. Luengnaruemitchai, S. Osuwan, and E. Gulari, Catal. Commun. 4 215 (2003).

${ }^{15}$ T. Tabakova, F. Boccuzzi, M. Manzoli, J. W. Sobczak, V. Idakiev, and D. Andreeva, Appl. Catal., B 49, 73 (2004).

${ }^{16}$ H. Sakurai, T. Akita, S. Tsubota, M. Kiuchi, and M. Haruta, Appl. Catal., A 291, 179 (2005).

${ }^{17}$ J. Guzman, S. Carrettin, J. C. Fierro-Gonzalez, Y. Hao, B. C. Gates, and A. Corma, Angew. Chem. Int. Ed. 44, 4778 (2005).

${ }^{18}$ R. Si and M. Flytzani-Stephanopoulos, Angew. Chem. Int. Ed. 47, 2884 (2008).

${ }^{19}$ W. Deng, A. I. Frenkel, R. Si, and M. Flytzani-Stephanopoulos, J. Phys. Chem. C 112, 12834 (2008).

${ }^{20}$ U. R. Pillai and S. Deevi, Appl. Catal., A 299, 266 (2006).

${ }^{21}$ M. Škoda, M. Cabala, I. Matolínová, K. C. Prince, T. Skála, F. Šutara, K. Veltruská, and V. Matolín, J. Chem. Phys. 130, 034703 (2009).

${ }^{22}$ J. A. Rodriguez, P. Liu, J. Hrbek, J. Evans, and M. Pérez, Angew. Chem. Int. Ed. 46, 1329 (2007).

${ }^{23}$ X. Wang, J. A. Rodriguez, J. C. Hanson, D. Gamarra, A. Martinez-Arias, and M. Fernández-García, J. Phys. Chem. B 110, 428 (2006).

${ }^{24}$ D. Tibiletti, A. Amieiro-Fonseca, R. Burch, Y. Chen, J. M. Fisher, A. Coguet, C. Hardacre, P. Hu, and D. Thompsett, J. Phys. Chem. B 109, 22553 (2005)

${ }^{25}$ X. Wang, J. A. Rodriguez, J. C. Hanson, M. Pérez, and J. Evans, J. Chem. Phys. 123, 221101 (2005).

${ }^{26}$ J. A. Rodriguez, S. Ma, P. Liu, J. Hrbek, J. Evans, and M. Pérez, Science 318, 1757 (2007)

${ }^{27}$ J.-L. Lu, H.-J. Gao, S. Shaikhutdinov, and H.-J. Freund, Catal. Lett. 114, 8 (2007).

${ }^{28}$ Z. P. Liu, S. J. Jenkins, and D. A. King, Phys. Rev. Lett. 94, 196102 (2005).

${ }^{29}$ C. Zhang, A. Michaelides, D. A. King, and S. J. Jenkins, J. Chem. Phys 129, 194708 (2008).

${ }^{30}$ N. C. Hernandez, R. Grau-Crespo, N. H. de Leeuw, and J. F. Sanz, Phys. Chem. Chem. Phys. 11, 5246 (2009).

${ }^{31}$ N. J. Castellani, M. M. Branda, K. M. Neyman, and F. Illas, J. Phys. Chem. C 113, 4948 (2009).

${ }^{32}$ Y. Chen, P. Hu, M.-H. Lee, and H. Wang, Surf. Sci. 602, 1736 (2008).

${ }^{33}$ I. de P. R. Moreira, F. Illas, and R. L. Martin, Phys. Rev. B 65, 155102 (2002).

${ }^{34}$ J. L. F. Da Silva, M. V. Ganduglia-Pirovano, J. Sauer, V. Bayer, and G. Kresse, Phys. Rev. B 75, 045121 (2007).
${ }^{35}$ P. J. Hay, R. L. Martin, J. Uddin, and G. E. Scuseria, J. Chem. Phys. 125, 034712 (2006).

${ }^{36}$ M. V. Ganduglia-Pirovano, A. Hofmann, and J. Sauer, Surf. Sci. Rep. 62, 219 (2007).

${ }^{37}$ M. V. Ganduglia-Pirovano, J. L. F. da Silva, and J. Sauer, Phys. Rev. Lett. 102, 026101 (2009).

${ }^{38}$ C. Franchini, V. Bayer, R. Podloucky, J. Paier, and G. Kresse, Phys. Rev. B 72, 045132 (2005).

${ }^{39}$ C. Franchini, R. Podloucky, J. Paier, M. Marsman, and G. Kresse, Phys. Rev. B 75, 195128 (2007).

${ }^{40}$ C. Franchini, R. Podloucky, F. Allegretti, F. Li, G. Parteder, S. Surnev, and F. P. Netzer, Phys. Rev. B 79, 035420 (2009).

${ }^{41}$ R. L. Martin and F. Illas, Phys. Rev. Lett. 79, 1539 (1997).

${ }^{42}$ V. I. Anisimov, F. Aryasetiawan, and A. I. Lichtenstein, J. Phys.: Condens. Matter 9, 767 (1997).

${ }^{43}$ V. I. Anisimov, I. V. Solovyev, M. A. Korotin, M. T. Czyzyk, and G. A. Sawatzky, Phys. Rev. B 48, 16929 (1993).

${ }^{44}$ I. V. Solovyev, P. H. Dederichs, and V. I. Anisimov, Phys. Rev. B 50, 16861 (1994).

${ }^{45}$ C. W. M. Castleton, J. Kullgren, and K. Hermansson, J. Chem. Phys. 127, 244704 (2007).

${ }^{46}$ M. Nolan, S. Grigoleit, D. C. Sayle, S. C. Parker, and G. W. Watson, Surf. Sci. 576, 217 (2005).

${ }^{47}$ C. Loschen, J. Carrasco, K. M. Neyman, and F. Illas, Phys. Rev. B 75, 035115 (2007).

${ }^{48}$ M. Cococcioni and S. de Gironcoli, Phys. Rev. B 71, 035105 (2005)

${ }^{49}$ S. J. Duclos, Y. K. Vohra, A. L. Ruoff, A. Jayaraman, and G. P. Espinosa, Phys. Rev. B 38, 7755 (1988).

${ }^{50}$ L. Gerward and J. S. Olsen, Powder Diffr. 8, 127 (1993).

${ }^{51}$ D. Muñoz, I. de P. R. Moreira, and F. Illas, Phys. Rev. Lett. 84, 1579 (2000).

${ }^{52}$ C. de Graaf, C. Sousa, I. de P. R. Moreira, and F. Illas, J. Phys. Chem. A 105, 11371 (2001)

${ }^{53}$ D. Muñoz, I. de P. R. Moreira, and F. Illas, Phys. Rev. B 71, 172505 (2005).

${ }^{54}$ J. Casanovas and F. Illas, J. Chem. Phys. 101, 7683 (1994).

${ }^{55}$ J. Casanovas, J. Rubio, and F. Illas, Phys. Rev. B 53, 945 (1996)

${ }^{56}$ M. M. Branda, C. Loschen, K. M. Neyman, and F. Illas, J. Phys. Chem. C 112, 17643 (2008).

${ }^{57}$ C. Loschen, S. T. Bromley, K. M. Neyman, and F. Illas, J. Phys. Chem. C 111, 10142 (2007).

${ }^{58}$ C. Loschen, A. Migani, S. T. Bromley, F. Illas, and K. M. Neyman, Phys. Chem. Chem. Phys. 10, 5730 (2008).

${ }^{59}$ A. Migani, C. Loschen, F. Illas, and K. M. Neyman, Chem. Phys. Lett. 465, 106 (2008).

${ }^{60}$ P. E. Blöchl, Phys. Rev. B 50, 17953 (1994).

${ }^{61}$ G. Kresse and D. Joubert, Phys. Rev. B 59, 1758 (1999).

${ }^{62}$ G. Kresse and J. Furthmüller, Phys. Rev. B 54, 11169 (1996)

${ }^{63}$ J. Heyd, G. E. Scuseria, and M. Ernzerhof, J. Chem. Phys. 118, 8207 (2003).

${ }^{64}$ J. Heyd, G. E. Scuseria, and M. Ernzerhof, J. Chem. Phys. 124, 219906 (2006).

${ }^{65}$ C. Adamo and V. Barone, J. Chem. Phys. 110, 6158 (1999).

${ }^{66}$ M. Ernzerhof and G. E. Scuseria, J. Chem. Phys. 110, 5029 (1999).

${ }^{67}$ J. P. Perdew, K. Burke, and M. Ernzerhof, Phys. Rev. Lett. 78, 1396 (1997).

${ }^{68}$ F. Corà, M. Alfredsson, G. Mallia, D. S. Middlemiss, W. C. Mackrodt, R. Dovesi, and R. Orlando, Struct. Bonding (Berlin) 113, 171 (2004).

${ }^{69}$ F. Illas and R. L. Martin, J. Chem. Phys. 108, 2519 (1998).

${ }^{70}$ D. Muñoz, N. M. Harrison, and F. Illas, Phys. Rev. B 69, 085115 (2004).

${ }^{71}$ R. Caballol, O. Castell, F. Illas, J. P. Malrieu, and I. de P. R. Moreira, J. Phys. Chem. A 101, 7860 (1997).

${ }^{72}$ F. Cora, Mol. Phys. 103, 2483 (2005).

${ }^{73}$ M. Alfredsson, G. D. Price, C. R. A. Catlow, S. C. Parker, R. Orlando, and J. P. Brodholt, Phys. Rev. B 70, 165111 (2004).

${ }^{74}$ I. Ciofini, F. Illas, and C. Adamo, J. Chem. Phys. 120, 3811 (2004).

${ }^{75}$ H. J. Monkhorst and J. D. Pack, Phys. Rev. B 13, 5188 (1976).

${ }^{76}$ R. F. W. Bader, Atoms in Molecules: A Quantum Theory (Oxford Science, Oxford, 1990)

${ }^{77}$ R. Grau-Crespo, N. C. Hernandez, J. F. Sanz, and N. H. de Leeuw, J. Mater. Chem. 19, 710 (2009).

${ }^{78}$ R. O. Jones and O. Gunnarsson, Rev. Mod. Phys. 61, 689 (1989).

${ }^{79}$ B. Hammer, L. B. Hansen, and J. K. Nørskov, Phys. Rev. B 59, 7413 (1999). 
${ }^{80}$ E. L. Wilson, R. Grau-Crespo, C. L. Pang, G. Cabailh, Q. Chen, C. R. A. Catlow, W. A. Brown, N. H. de Leeuw, and G. Thornton, J. Phys. Chem. C 112, 10918 (2008).

${ }^{81}$ R. Grau-Crespo, F. Cora, A. A. Sokol, N. H. de Leeuw, and C. R. A. Catlow, Phys. Rev. B 73, 035116 (2006).
${ }^{82}$ R. Grau-Crespo, N. C. Hernandez, J. F. Sanz, and N. H. de Leeuw, J. Phys. Chem. C 111, 10448 (2007).

${ }^{83}$ J. C. Wojdeł, I. de P. R. Moreira, and F. Illas, J. Am. Chem. Soc. 131, 906 (2009). 